\title{
Full-wave analysis of a two slot microstrip filter using a new algorithm for computation
} of the spectral integrals

\section{Gothelf, Ulrich V.; Østergaard, Erik Allan}

Published in:

I E E E Transactions on Microwave Theory and Techniques

Link to article, DOI:

$10.1109 / 22.210235$

Publication date:

1993

Document Version

Publisher's PDF, also known as Version of record

Link back to DTU Orbit

Citation (APA):

Gothelf, U. V., \& Østergaard, E. A. (1993). Full-wave analysis of a two slot microstrip filter using a new algorithm for computation of the spectral integrals. I E E E Transactions on Microwave Theory and Techniques, 41(1), 101-108. https://doi.org/10.1109/22.210235

\section{General rights}

Copyright and moral rights for the publications made accessible in the public portal are retained by the authors and/or other copyright owners and it is a condition of accessing publications that users recognise and abide by the legal requirements associated with these rights.

- Users may download and print one copy of any publication from the public portal for the purpose of private study or research.

- You may not further distribute the material or use it for any profit-making activity or commercial gain

- You may freely distribute the URL identifying the publication in the public portal 


\section{Full-Wave Analysis of a Two Slot Microstrip Filter Using a New Algorithm for Computation of the Spectral Integrals}

Ulrich V. Gothelf, Student Member, IEEE, and Allan Østergaard, Member, IEEE

\begin{abstract}
An integral equation is formulated in the spectral domain for a two slot microstrip filter using the exact Green's function for the grounded dielectric substrate. Using a moment method (MM) procedure, the integral equation has been discretized. The elements of the impedance matrix and the excitation vector are given by two dimensional Sommerfeld type integrals in closed form. An efficient and accurate numerical integration scheme for computation of the elements is presented. The $S$ parameters obtained from the MM procedure have been found to be in excellent agreement with measurements.
\end{abstract}

\section{INTRODUCTION}

$\mathbf{I}$ $\mathrm{N}$ THE PRESENT communication we consider the microstrip filter problem shown in Fig. 1. An integral equation for the filter problem is derived using the exact Green's function for the geometry. Hence, both free space radiation and surface waves are included in the solution. Using basis functions modeling the incident, reflected and transmitted currents on the filter [1], the integral equation is solved numerically for the scattering parameters using a Galerkin type MM procedure.

The elements of the impedance matrix and source vector obtained from the MM procedure are given by two dimensional integrals over the spectral coordinates $k_{x}$ and $k_{y}$.

Instead of integrating these integrals in the polar coordinate system [1], an efficient and accurate scheme is applied in which the integrals are integrated in the cartesian $k_{x}$ and $k_{y}$ coordinates using a deformation technique in which the integration contour follows a path parallel to the imaginary axis in the complex plane for one of the integration variables [2] while the other integration variable is integrated along the real axis using the weighted average algorithm [3]. In this communication no approximation for the Green's function is used nor are the integration limits truncated.

In order to test the integration algorithm, the microstrip filter problem was chosen since the space radiation and surface wave radiation at resonance were expected to be substantial, hence the transfer function of the filter would be suited for testing against precision measurements using a HP8510 network analyzer.

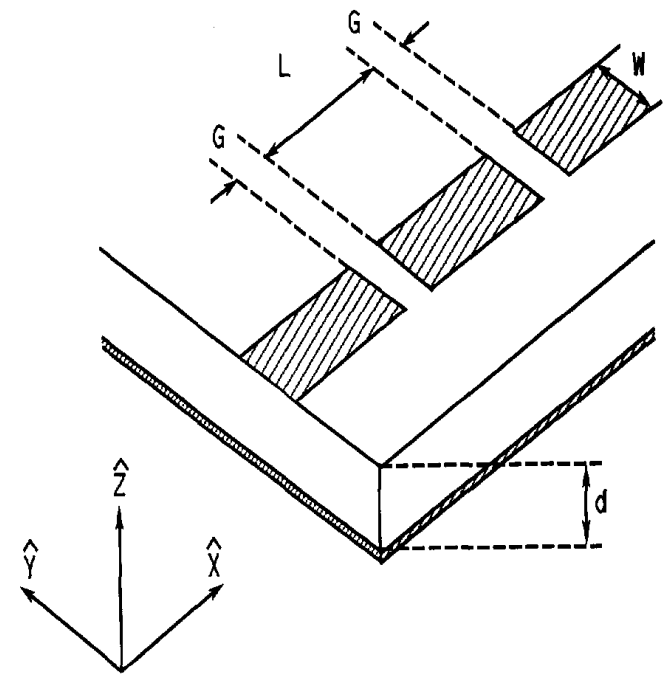

Fig. 1. Geometry of microstrip filter.

\section{THEORY}

In Fig. 1, the outline of the microstrip filter is shown. The filter is in the form of an infinite microstrip line in which two narrow gaps are cut. The substrate has thickness $d$, relative permittivity $\epsilon_{r}$ and is assumed to be infinitely wide in the $x$ and $y$ directions. The filter lines are assumed to be infinitely thin and perfectly conducting. Since the width of the filter lines are narrow in terms of wavelength, we consider only $x$ directed surface currents. [4]

Green's Function for the Grounded Dielectric Slab:

Using the spectral domain Green's function, we obtain the $\mathrm{x}$-directed electric field at $(x, y, d)$ from an $x$-directed current distribution [5], [6]:

$$
\begin{aligned}
& E_{x x}(x, y, d) \\
& =\frac{1}{4 \pi^{2}} \iint_{-\infty}^{\infty} \tilde{G}_{x x}\left(k_{x}, k_{y}\right) \tilde{K}_{x}\left(k_{x}, k_{y}\right) e^{j k_{x} x} e^{j k_{y} y} d k_{x} d k_{y}
\end{aligned}
$$

where

$$
\tilde{K}_{x}\left(k_{x}, k_{y}\right)=\iint_{-\infty}^{\infty} K_{x}(x, y, d) e^{-j k_{x} x} e^{-j k_{y} y} d x d y
$$

Manuscript received September 17, 1991; revised April 9, 1992. of Denmark, Bldg. 348, OK-2800 Lyngby, Denmark.

IEEE Log Number 9204024. 
$K_{x}(x, y, d)$ is the $x$-directed electric surface current distribution on the microstrip filter lines.

$$
\begin{aligned}
\tilde{G}_{x x}= & -\frac{j \eta}{k_{0}} \\
& \cdot \frac{\left(\epsilon_{r} k_{0}^{2}-k_{x}^{2}\right) k_{z 2} \cos \left(k_{z 1} d\right)+j\left(k_{o}^{2}-k_{x}^{2}\right) k_{z 1} \sin \left(k_{z 1} d\right)}{T_{e} T_{m}} \\
& \cdot \sin \left(k_{z 1} d\right), k_{0}=\omega \sqrt{\mu \epsilon} \\
T_{m} & =\epsilon_{r} k_{z 2} \cos \left(k_{z 1} d\right)+j k_{z 1} \sin \left(k_{z 1} d\right) \\
T_{e} & =k_{z 1} \cos \left(k_{z 1} d\right)+j k_{z 2} \sin \left(k_{z 1} d\right) \\
k_{z 1}^{2} & =\epsilon_{r} k_{0}^{2}-\lambda^{2} \quad \operatorname{Im}\left(k_{z 1}\right)<0 \\
k_{z 2}^{2} & =k_{0}^{2}-\lambda^{2} \\
\lambda^{2} & =k_{x}^{2}+k_{y}^{2} .
\end{aligned}
$$

\section{Infinite Microstrip Line}

An accurate solution of the microstrip filter problem requires that the effective propagation constant $k_{e}$ for the current distribution on the half infinite microstrip lines is computed accurately. The effective propagation constant is computed from the corresponding infinite microstrip line problem where we have assumed the traveling-wave form $e^{-j k_{e} x}[1]$ for the $x$-dependence of the $x$-directed current distribution.

Since the main computational effort is spent computing the impedance matrix elements obtained from the Galerkin procedure, a judicious choice of basis functions is important to obtain a convergent solution using a minimal number of basis functions. The $y$-dependence of the current distribution is expanded in a set of entire domain Maxwellian basis functions (10) which are also used as test functions [7, p. 342]. The Maxwellian functions satisfy the proper edge condition for the surface current and are even functions in the transverse coordinate $y$, as required by the symmetry of the geometry.

The method of solution involves formulating an integral equation for the $x$-directed electric field on the microstrip line which is discretized using the Galerkin's method of moment procedure. The linear system obtained is subsequently solved for the unknown expansion coefficients:

$$
\begin{aligned}
K_{x}(x, y) & =K_{x}(x) K_{x}(y) \\
K_{x}(y) & =\sum_{q=1}^{N_{y}} C_{q} f_{y q}(y) \\
K_{x}(x) & =e^{-j k_{e} x}
\end{aligned}
$$

where

$$
f_{y q}(y)=\frac{\cos \left(y(q-1) \frac{2 \pi}{w}\right)}{\sqrt{1-\left(y \frac{2}{w}\right)^{2}}} ; \quad q=1,2, \cdots .
$$

In the spectral domain, the basis functions are given by

$$
\begin{aligned}
\tilde{f}_{y q}\left(k_{y}\right)=\frac{w \pi}{4} & \left(J_{0}\left(k_{y} \frac{w}{2}+(q-1) \pi\right)\right. \\
& \left.+J_{0}\left(k_{y} \frac{w}{2}-(q-1) \pi\right)\right) ; \quad q=1,2, \cdots .
\end{aligned}
$$

Because of the $x$-dependence of the $\mathrm{x}$-directed current distribution (1) is reduced to (12), [1]:

$$
E_{x x}(x, y, d)=\frac{1}{4 \pi^{2}} \int_{-\infty}^{\infty} \tilde{G}_{x x}\left(k_{e}, k_{y}\right) \tilde{K}_{x}\left(k_{y}\right) e^{j k_{y y}} d k_{y}
$$

Applying Galerkin's method of moments on (12) yields

$$
\left[\begin{array}{lll}
Z_{11} & Z_{12} & Z_{13} \\
Z_{21} & Z_{22} & Z_{23} \\
Z_{31} & Z_{32} & Z_{33}
\end{array}\right] \cdot\left[\begin{array}{l}
C_{1} \\
C_{2} \\
C_{3}
\end{array}\right]=\left[\begin{array}{l}
0 \\
0 \\
0
\end{array}\right]
$$

where

$$
Z_{p q}=\frac{1}{2 \pi^{2}} \int_{0}^{\infty} \tilde{G}_{x x}\left(k_{e}, k_{y}\right) \tilde{f}_{y q}\left(k_{y}\right) \tilde{f}_{y p}^{*}\left(k_{y}\right) d k_{y} .
$$

Since $k_{e}$ is found to be larger than the surface wave poles in the Green's function, all elements in (14) are pure imaginary [1], [8]. Hence (13) can be converted to a real matrix equation. For the proper value of $k_{e}$, the determinant of the $Z$ matrix in (13) is zero. Starting with an assumed value of $k_{e}$, the secant root seeking method is used for obtaining the true value of $k_{e}$. When $k_{e}$ is determined, the $C_{n}$ coefficients are obtained as an eigenvector to the Z-matrix in (13) [7, p. 342].

The $C_{n}$ coefficients given by the eigenvector $\left(C_{1}=1\right)$ are subsequently used for the incident, reflected and transmitted waves on the half infinite microstrip lines.

\section{Microstrip Filter}

Having determined the effective propagation constant and the $C_{n}$ coefficients for the $\mathrm{y}$-dependence of the current distribution on the infinite microstrip line, we define an incident, a reflected and a transmitted electric current. Using $R$ and $T$ for the reflection and transmission coefficients respectively, the $x$ - and $y$-dependence of the incident, reflected and transmitted currents [1] may be written in the form (17):

$$
K_{i n c}+K_{r e f}=\left((1-R) f_{s 1}(x)-j(1+R) f_{s 2}(x)\right) f(y)
$$

where

$$
\begin{gathered}
f_{s 1}(x)=f_{s}\left(k_{e} x+\frac{\pi}{2}\right), \quad f_{s 2}(x)=f_{s}\left(k_{e} x\right) \\
f_{s}(u)=\left\{\begin{array}{cl}
\sin u & -M \pi<u<0 \\
0 & \text { otherwise }
\end{array}\right\}
\end{gathered}
$$

and

$$
K_{t r}=\left(-T f_{u 1}(x)-j T f_{u 2}(x)\right) f(y)
$$

where

$$
\begin{aligned}
f_{u 1}(x) & =f_{u}\left(k_{e}(x-L-2 G)-\frac{\pi}{2}\right) \\
; f_{u 2}(x) & =f_{u}\left(k_{e}(x-L-2 G)\right) \\
f_{u}(u) & =\left\{\begin{array}{cl}
\sin u & 0<u<M \pi \\
0 & \text { otherwise }
\end{array}\right\} \\
f(y) & =\sum_{q=1}^{N_{3}} C_{q} f_{y q}(y), C_{1}=1 .
\end{aligned}
$$




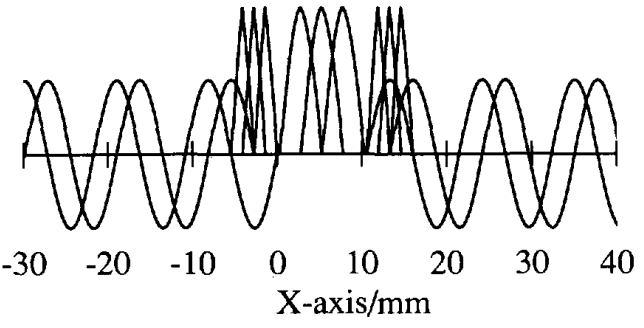

Fig. 2. Expansion modes on the microstrip filter.

Whether $M$ in (19) and (22) is an integer value or not is of no consequence for the numerical convergence of the corresponding integrals obtained later from the Galerkin procedure when computed by the integration scheme described in this paper. However, $M$ is chosen as an integer since this yields a simpler expression in the spectral domain. Numerical tests indicate that the solution to the filter problem is almost independent of $M$ when the traveling wave modes are longer than five wavelengths $(M=10)$. For the computations in the present paper, we have used $M=15$.

Subsectional piecewise sinusoidal (PWS) modes have been used for modeling the $\mathrm{x}$-dependence of the current in the vicinity of the gaps as shown in Fig. 2. The PWS modes are defined

$$
f_{x n}(x)=\left\{\begin{array}{cc}
\frac{\sin k_{e}\left(h_{n}-\left|x-x_{n}\right|\right)}{\sin k_{e} h_{n}} & {\left[x-x_{n}\right] \leq h_{n}} \\
0 & {\left[x-x_{n}\right]>h_{n}}
\end{array}\right\}
$$

where $2 h_{n}$ is the width and $x_{n}$ is the position of the centre of the $n$th PWS function. The $y$-dependence of the $N_{x} /, x$-directed PWS modes are expanded into a sum of $N_{y}$ Maxwellian modes (10).

Substituting the true current with an expansion using the basis functions described, the $\mathrm{x}$-directed E-field at the upper surface of the dielectric layer may be written in the form of an integral equation (25):

$$
\begin{aligned}
& E_{x}(x, y, d)= \\
& \iint_{-\infty}^{\infty} \tilde{G}_{x x}\left(\tilde{K}_{i n c}+\tilde{K}_{r e f}+\tilde{K}_{t r}+\sum_{q=1}^{N_{y}} \sum_{n=1}^{N_{x}} K_{n q} \tilde{f}_{x n} \tilde{f}_{y q}\right) \\
& \cdot e^{j k_{x} x} e^{j k_{y} y} d k_{x} d k_{y} .
\end{aligned}
$$

Equation (25) is tested with $\left(N_{x}+2\right) N_{y}$ PWS test functions (10), (24). Since the traveling wave modes are obtained from the solution of the infinite microstrip line problem, the boundary condition for the tangential electric field is automatically satisfied away from the gaps. Hence, it is only necessary to test (25) in the gap regions.

The PWS test functions are located as the PWS basis functions are, except for the two additional test functions necessary to obtain the same number of equations as unknowns. The two additional test PWS functions are located immediately after the last PWS basis function at each side of the two gaps. The y-dependence $K_{x}(y)$ of these two test functions are assumed identical to the traveling wave modes.

The impedance matrix elements can then be defined using the following indices:

$n \quad$ Index of PWS basis functions $x$-dependent part

$q$ Index of PWS basis functions $y$-dependent part

$m$ Index of PWS test functions $x$-dependent part

$p$ Index of PWS test functions $y$-dependent part

$t 1$ Additional test functions on the left microstrip line

$t 2$ Additional test functions on the right microstrip line.

The testing procedure results in a matrix equation for the unknown coefficients $R, T, K_{11}, \cdots, K_{n q}, \cdots, K_{N_{x} N_{y}}$.

A simple example with two PWS modes on the centre strip (and none on each of the half infinite lines), each expanded in two Maxwellian modes for the y-dependence, yields matrix equation (31), which is shown at the bottom of the next page.

$$
\begin{aligned}
Z_{m n p q} & =\iint_{-\infty}^{\infty} \tilde{G}_{x x} \tilde{f}_{x n} \tilde{f}_{x m}^{*} \tilde{f}_{y q} \tilde{f}_{y p}^{*} d k_{x} d k_{y} \\
Z_{m p s i} & =\iint_{-\infty}^{\infty} \tilde{G}_{x x} \tilde{f}_{s i} \tilde{f}_{x m}^{*} \tilde{f}_{y} \tilde{f}_{y p}^{*} d k_{x} d k_{y}=\sum_{q=1}^{N_{y}} C_{q} \iint_{-\infty}^{\infty} \tilde{G}_{x x} \tilde{f}_{s i} \tilde{f}_{x m}^{*} \tilde{f}_{y q} \tilde{f}_{y p}^{*} d k_{x} d k_{y} \quad i \in\{1,2\} \\
Z_{m p u l} & =\iint_{-\infty}^{\infty} \tilde{G}_{x x} \tilde{f}_{u i} \tilde{f}_{x m}^{*} \tilde{f}_{y} \tilde{f}_{y p}^{*} d k_{x} d k_{y}=\sum_{q=1}^{N_{y}} C_{q} \iint_{-\infty}^{\infty} \tilde{G}_{x x} \tilde{f}_{u i} \tilde{f}_{x m}^{*} \tilde{f}_{y q} \tilde{f}_{y p}^{*} d k_{x} d k_{y} \quad i \in\{1,2\} \\
Z_{t i n q} & =\iint_{-\infty}^{\infty} \tilde{G}_{x x} \tilde{f}_{x n} \tilde{f}_{x\left(N_{x}+i\right)} \tilde{f}_{y q} \tilde{f}_{y}^{*} d k_{x} d k_{y}=\sum_{p=1}^{N_{y}} C_{p} \iint_{-\infty}^{\infty} \tilde{G}_{x x} \tilde{f}_{x n} \tilde{f}_{x\left(N_{x}+i\right)}^{*} \tilde{f}_{y q} \tilde{f}_{y p}^{*} d k_{x} d k_{y} \quad t \in\{1,2\} \\
Z_{t i l j}= & \iint_{-\infty}^{\infty} \tilde{G}_{x x} \tilde{f}_{l j} \tilde{f}_{x\left(N_{x}+i\right)}^{*} \tilde{f}_{y} \tilde{f}_{y}^{*} d k_{x} d k_{y} \\
& =\sum_{q=1}^{N_{y}} \sum_{p=1}^{N_{y}} C_{q} C_{p} \iint_{-\infty}^{\infty} \tilde{G}_{x x} \tilde{f}_{l j} \tilde{f}_{x\left(N_{x}+i\right)}^{*} \tilde{f}_{y q} \tilde{f}_{y p}^{*} d k_{x} d k_{y} \quad i, j \in\{1,2\} l \in\{s, u\}
\end{aligned}
$$




\section{COMPUTATION OF THE Z AND THE V ElEmentS}

The main computational effort when solving the filter problem is the numerical computation of the individual elements in the impedance matrix $Z$ and the excitation vector $V$. The elements are given by two dimensional Sommerfeld type integrals given in closed form in (26) to (30), which is shown at the bottom of the previous page. The integrands contain poles corresponding to the generation of surface waves. Furthermore, the integrands are rapidly oscillating as $k_{x}^{2}+k_{y}^{2} \rightarrow \infty$, especially as the distance between basis and test functions increases.

Usually, the spectral domain integration intervals in (26) to (30) are transformed into finite and semi-infinite intervals by changing the cartesian integration variables $\left(k_{x}, k_{y}\right)$ into the polar integration variables $(\lambda, \phi)[3],[8],[9]$.

One difficulty when using the polar integration scheme is to compute the $\phi$ integration accurately since the oscillation frequency of the integrand is directly proportional to the integration variable $\lambda$. Attempting to truncate the semi-infinite integration interval for $\lambda$ in order to set an upper bound for the oscillations in $\phi$ reduces the obtainable numerical accuracy of the integral which is slowly convergent.

In this work, the numerical computation of the integrals is made in the cartesian coordinates $\left(k_{x}, k_{y}\right)$ without truncation of the infinite intervals.

Since $\tilde{f}_{a}$ and $\tilde{f}_{b}$ are real functions and $\tilde{G}_{x x}$ is an even function of $k_{x}$ and of $k_{y}$ the integrals in (26) to (30) can be written as in (32):

$$
\begin{aligned}
Z & =\int_{-\infty}^{\infty} \tilde{S}\left(k_{y}\right) \tilde{f}_{a}\left(k_{y}\right) \tilde{f}_{b}^{*}\left(k_{y}\right) d k_{y} \\
& =\int_{0}^{\infty} \tilde{S}\left(k_{y}\right)\left(\tilde{f}_{a}\left(k_{y}\right) \tilde{f}_{b}^{*}\left(k_{y}\right)+\tilde{f}_{a}^{*}\left(k_{y}\right) \tilde{f}_{b}\left(k_{y}\right)\right) d k_{y}
\end{aligned}
$$

where

$$
\begin{aligned}
\tilde{S}\left(k_{y}\right) & =\int_{-\infty}^{\infty} \tilde{G}_{x x}\left(k_{x}, k_{y}\right) \tilde{f}_{a}\left(k_{x}\right) \tilde{f}_{b}^{*}\left(k_{x}\right) d k_{x} \\
& =\int_{0}^{\infty} \tilde{G}_{x x}\left(k_{x}, k_{y}\right)\left(\tilde{f}_{a}\left(k_{x}\right) \tilde{f}_{b}^{*}\left(k_{x}\right)+\tilde{f}_{a}^{*}\left(k_{x}\right) \tilde{f}_{b}\left(k_{s}\right)\right) d k_{x} .
\end{aligned}
$$

The singularities of the Green's function are located in the range $k_{0}^{2}<k_{x}^{2}+k_{y}^{2}<\epsilon_{r} k_{0}^{2}[11]$ and correspond to transverse magnetic surface waves $T_{m}(\lambda)=0$ and/or transverse electric surface waves $T_{e}(\lambda)=0$. In the $\left(k_{x}, k_{y}\right)$ plane, the poles are located on concentric circles. Hence, when integrating along a contour parallel to the $k_{y}$ axis, the position of singular points (if any) depends on the value of $k_{x}$. In order to avoid the difficulty of $k_{x}\left(k_{y}\right)$-dependent singular points when

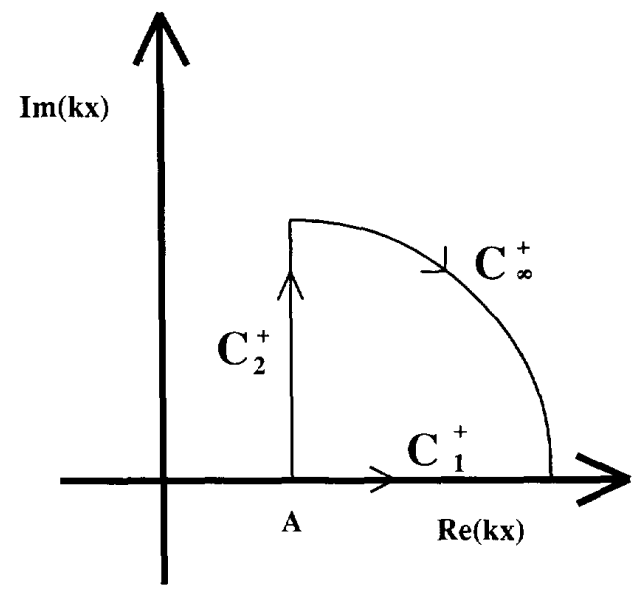

Fig. 3. Integration contour used in the complex $k_{x}$ and $k_{y}$ planes.

integrating parallel to the $k_{y}\left(k_{x}\right)$ axis, the integration path has been deformed into the complex $k_{y}\left(k_{x}\right)$ plane in the range from 0 to $\left(1+\sqrt{\epsilon_{r}}\right) k_{0}$ using the Cauchy integration theorem [12]. The deformed integration path used for both the $k_{x}$ and the $k_{y}$ integration variables is shown in Fig. 3.

Alternative integration contours have been investigated. Rexberg [9] uses the upper half of an ellipse for the integration path in the $\lambda$ plane. In [2a], a triangle is used for the integration contour in both the $k_{x}$ and the $k_{y}$ planes.

The integration path cannot be arbitrarily deformed into the first quadrant for the $k_{x}$ variable when using the approach described in the present paper since the integration contour chosen later for the $k_{y}$ variable introduces branch cuts in this quadrant. Furthermore, care must be taken when choosing the path for numerical reasons. If the path is too close to the singular points on the real axis, the values of the integrand become too large for accurate numerical computation. On the other hand, if the integration path is too far from the real axis, the integrand increases exponentially in amplitude.

It is necessary to integrate the three linear parts of which the deformed contour is composed independently since the path itself is not differentiable. In spite of this, we found from numerical experiments that the integration contour in Fig. 3 was more efficient in terms of integration points for a given accuracy than the elliptic path in the complex $k_{x}$ and $k_{y}$ planes. Furthermore, we found that the optimum values for $\operatorname{Im}\left(k_{x}\right)$ and $\operatorname{Im}\left(k_{y}\right)$ to be about $0.1 k_{0}$, which is similar to the value choose by Newman and Forrai [10] for the deformation into the complex $\lambda$ plane.

$$
\left[\begin{array}{cccccc}
Z_{1111} & Z_{1112} & Z_{1211} & Z_{1212} & \left(-Z_{11 s 1}-j Z_{11 s 2}\right) & \left(-Z_{11 u 1}-j Z_{11 u 2}\right) \\
Z_{1121} & Z_{1122} & Z_{1221} & Z_{1222} & \left(-Z_{12 s 1}-j Z_{12 s 2}\right) & \left(-Z_{12 u 1}-j Z_{12 u 2}\right) \\
Z_{2111} & Z_{2112} & Z_{2211} & Z_{2212} & \left(-Z_{21 s 1}-j Z_{21 s 2}\right) & \left(-Z_{21 u 1}-j Z_{21 u 2}\right) \\
Z_{2121} & Z_{2122} & Z_{2221} & Z_{2222} & \left(-Z_{22 s 1}-j Z_{22 s 2}\right) & \left(-Z_{22 u 1}-j Z_{22 u 2}\right) \\
Z_{t 111} & Z_{t 112} & Z_{t 121} & Z_{t 122} & \left(-Z_{t 1 s 1}-j Z_{t 1 s 2}\right) & \left(-Z_{t 1 u 1}-j Z_{t 1 u 2}\right) \\
Z_{t 211} & Z_{t 112} & Z_{t 121} & Z_{t 122} & \left(-Z_{t 2 s 1}-j Z_{t 2 s 2}\right) & \left(-Z_{t 2 u 1}-j Z_{t 2 u 2}\right)
\end{array}\right] \cdot\left[\begin{array}{c}
K_{11} \\
K_{12} \\
K_{21} \\
K_{22} \\
R \\
T
\end{array}\right]=\left[\begin{array}{c}
-Z_{11 s 1}+j Z_{11 s 2} \\
-Z_{12 s 1}+j Z_{12 s 2} \\
-Z_{21 s 1}+j Z_{21 s 2} \\
-Z_{22 s 1}+j Z_{22 s 2} \\
-Z_{t 1 s 1}+j Z_{t 1 s 2} \\
-Z_{t 2 s 1}+j Z_{t 2 s 2}
\end{array}\right]
$$


It is important to note that the integrand must be analytical when the Cauchy integration theorem is used. Hence, the complex conjugated terms in (32) and (33) must be computed as if $k_{x}$ and $k_{y}$ where pure real because the $k_{x}^{*}$ and $k_{y}^{*}$ terms are not analytical.

The $k_{y}$ integration of (32) in the interval from $\left(\sqrt{\epsilon_{r}}+1\right) k_{0}$ to $k_{\text {asymp }}=45 / W$ is made using the Gauss-Legendre integration algorithm. For $k_{y}$ larger than $k_{a s y m p}$, the product between the basis and test functions in (32) is written as a sum of functions (34) each composed of an oscillating and a nonoscillating part given in closed form using the Jacobi asymptotic expansion [13] for the zero order Bessel functions.

$$
\tilde{f}_{a}\left(k_{y}\right) \tilde{f}_{b}^{*}\left(k_{y}\right)+\tilde{f}_{a}^{*}\left(k_{y}\right) \tilde{f}_{b}\left(k_{y}\right)=\sum_{i} \tilde{A}_{i}\left(k_{y}\right) \cos \left(\omega_{i} k_{y}\right)
$$

For the Maxwellian basis and test functions used for the transverse dependence of the microstrip line current, the right hand side of (34) contains two terms. One term has twice the oscillation frequency of the Bessel functions while the other term becomes nonoscillating. The integration of the nonoscillating part is made using the Gauss-Legendre integration algorithm after applying the variable transformation (35):

$$
\begin{gathered}
v=\frac{1}{k_{y}} \quad d k_{y}=-\frac{1}{v^{2}} d v \\
\int_{A}^{\infty} \tilde{G}_{x x}\left(k_{x}, k_{y}\right) \tilde{A}_{i}\left(k_{y}\right) d k_{y} \\
=\int_{0}^{1 / A} \tilde{G}_{x x}\left(k_{x}, \frac{1}{v}\right) \tilde{A}_{i}\left(\frac{1}{v}\right) \frac{1}{v^{2}} d v ; \quad \text { for } \omega_{i}=0
\end{gathered}
$$

Distribution of Integration Points: Integration of the oscillating part is made using Mosig's weighted average algorithm [3]. We have used $25 k_{y}$ integration points for each of the sections of the integration contour above the branch point and surface wave poles. In the interval from $\left(\sqrt{\epsilon_{r}}+1\right) k_{0}$ to $k_{a s y m p}, 100 k_{y}$ integration points have been used. Twelve integration points have been used for the nonoscillating integral in (36) while 24 point have been used with the weighted average algorithm (4 half periods each determined using 6 integration points) for the oscillating part.

The $(25+25+25+100+12+24)=211$ integration points used for the $k_{y}$ integration determines $\tilde{S}\left(k_{x}\right)$ with 6 significant digits.

$\tilde{S}\left(k_{x}\right)$ in $(33)$ is tabulated for all the values of $k_{x}$ used later with the numerical integration in the $k_{x}$ direction. The integral (32) is calculated using an identical integration contour for the $k_{y}$ integration above the branch point and surface wave poles.

The $k_{x}$ integration from $k_{x}=\left(\sqrt{\epsilon_{r}}+1\right) k_{0}$ to infinity is determined using deformation of the integration contour parallel to the imaginary axis [12].

Yang [2a] has applied this technique for rooftop basis functions and an asymptotic approximation for the Green's function.

In this work no approximations with respect to the Green's functions are used. Furthermore, our approach is general in the sense that the impedance matrix elements can be computed regardless of the basis and test functions chosen.
The weighted average algorithm is less efficient for the $k_{x}$ integration when compared to the deformation technique since the contribution from each of the oscillating terms in (34) must be computed independently.

The combination of basis and test functions is rewritten into a sum of amplitude functions multiplied by exponential functions with pure imaginary arguments:

$$
\tilde{f}_{a}\left(k_{x}\right) \tilde{f}_{b}^{*}\left(k_{x}\right)+\tilde{f}_{a}^{*}\left(k_{x}\right) \tilde{f}_{b}\left(k_{x}\right)=\sum_{i} \tilde{A}_{i}\left(k_{x}\right) e^{j \omega_{i} k_{x}} ; \omega_{i} \in R .
$$

From (32) and (37) we obtain

$$
\begin{aligned}
Z= & \int_{0}^{\left(\sqrt{\epsilon_{r}}+1\right) k_{0}} \tilde{S}\left(k_{x}\right)\left(\tilde{f}_{a}\left(k_{x}\right) \tilde{f}_{b}^{*}\left(k_{x}\right)+\tilde{f}_{a}^{*}\left(k_{x}\right) \tilde{f}_{b}\left(k_{x}\right)\right) d k_{x} \\
& +\sum_{i} Z_{i}
\end{aligned}
$$

where

$$
Z_{i}=\int_{\left(\sqrt{\epsilon_{r}}+1\right) k_{0}}^{\infty} \tilde{S}\left(k_{x}\right) \tilde{A}_{i}\left(k_{x}\right) e^{j \omega_{i} k_{x}} d k_{x}
$$

It is important that all the integrands are analytical and that the integration contours do not cross any branch cuts. These conditions are met for the integrand in (39). In Fig. 4, it is shown how the deformation and the transformation is made for $\omega_{i}>0$.

We have proved that the integration contour along $C_{\infty}^{+}$does not contribute to $Z_{i}$ (39). Hence the integrals along $C_{1}^{+}$and $\mathrm{C}_{2}^{+}$are identical.

$$
Z_{i}=\int_{C_{2}^{+}} \tilde{S}\left(k_{x}\right) \tilde{A}_{i}\left(k_{x}\right) e^{j \omega_{i} k_{x}} d k_{x}
$$

We define

$$
\tilde{F}_{i}\left(k_{x}\right)=\tilde{S}\left(k_{x}\right) \tilde{A}_{i}\left(k_{x}\right)
$$

The path $C_{2}^{+}$is given by

$$
\begin{aligned}
& k_{x}=A+j t \quad \frac{d k_{x}}{d t}=j \\
& t \in[0, \infty] \quad \text { where } A=\left(\sqrt{\epsilon_{r}}+1\right) k_{0} .
\end{aligned}
$$

From (40), (41) and (42) we obtain

$$
\begin{aligned}
Z_{i} & =\int_{0}^{\infty} \tilde{F}_{i}(A+j t) e^{j(A+j t) \omega_{i}} j d t \\
& =j e^{j A \omega_{i}} \int_{0}^{\infty} \tilde{F}_{i}(A+j t) e^{-\omega_{i} t} d t \quad \omega_{i}>0 .
\end{aligned}
$$

Since the integrand in (43) is decreasing exponentially, the variable transformation (44) yielding a finite integration interval can be applied without introducing numerial difficulties.

$$
t=-\frac{1}{g} \log z ; \quad \frac{d t}{d z}=-\frac{1}{g z} ; \quad g>0 .
$$

Using (44) upon (43) we obtain

$$
Z_{i}=j e^{j A \omega_{i}} \int_{0}^{1} \tilde{F}_{i}\left(A-j \frac{1}{g} \log z\right) z^{\omega_{i} / g-1} d z \quad \omega_{i}>0 .
$$

For $\omega_{i}<0$ a similar procedure is used. For $\omega_{i}=0$ the variable transformation (35) is applied for the $k_{x}$ variable. 


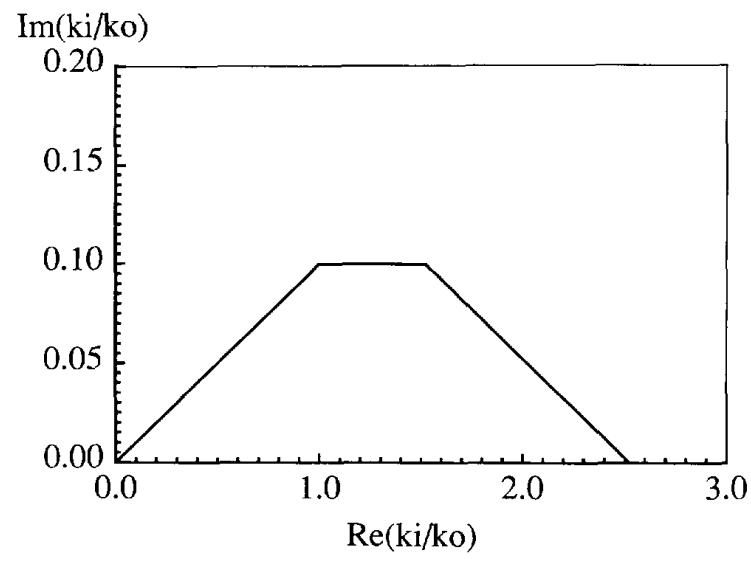

Fig. 4. Deformation of integration contour.

In summary, see (46), which is at the bottom of this page. In (44) we have introduced a constant $g>0$. The value of (46) is independent of $g$. However, the location and the number of integration points necessary for the numerical computation of the integral in (46), given a certain accuracy, is dependent upon the actual value of $g$. Hence, the optimum, in terms of the computational effort, is to minimize the sum of the number of integration points used with the selected values of $g$.

In all the integrals for the filter, we only use $\tilde{G}_{x x}$ from the dyadic Green's function, however the integration scheme described in this paper can be applied for any of the remaining elements in the dyadic Green's function.

Since $\tilde{G}_{x x}$ is an even function of $k_{z 1}$, one may ignore the branch cut due to $k_{z 1}=\sqrt{\epsilon_{\mathrm{r}} k_{0}^{2}-k_{x}^{2}-k_{y}^{2}}$. The branch cut due to $k_{z 2}=\sqrt{k_{0}^{2}-k_{x}^{2}-k_{y}^{2}}$ which applies for $\tilde{G}_{x x}$ is shown in Fig. 5. From Fig. 5 we appreciate that either the $k_{x}$ or the $k_{y}$ integration must be performed along a contour parallel to the real axis.

For the $k_{x}$ integration, $(25+25+25+12+2 \cdot 50)=187$ integrations points are used. The first 75 points are used for the deformation contours above the surface wave poles. The 12 integration points are used for the nonoscillating part in (46). The remaining $2 * 50$ points are used for the deformation into the first and fourth quadrants of the complex plane.

The accuracy obtained for the elements of $Z$ and $V$ are 4 to 5 significant digits regardless of the distance between the basis and test functions. The accuracy of the current vector (31) is also 4 to 5 digit.

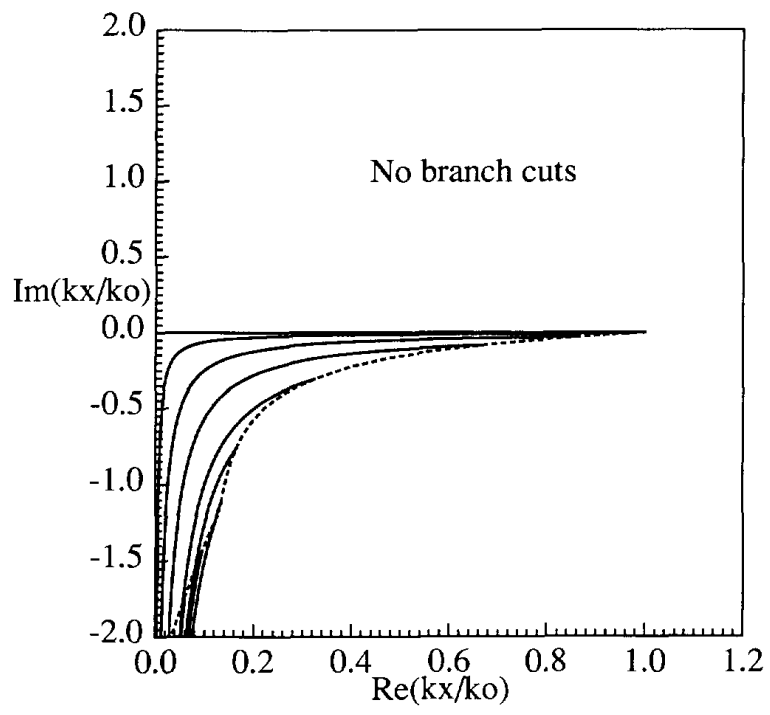

(a)

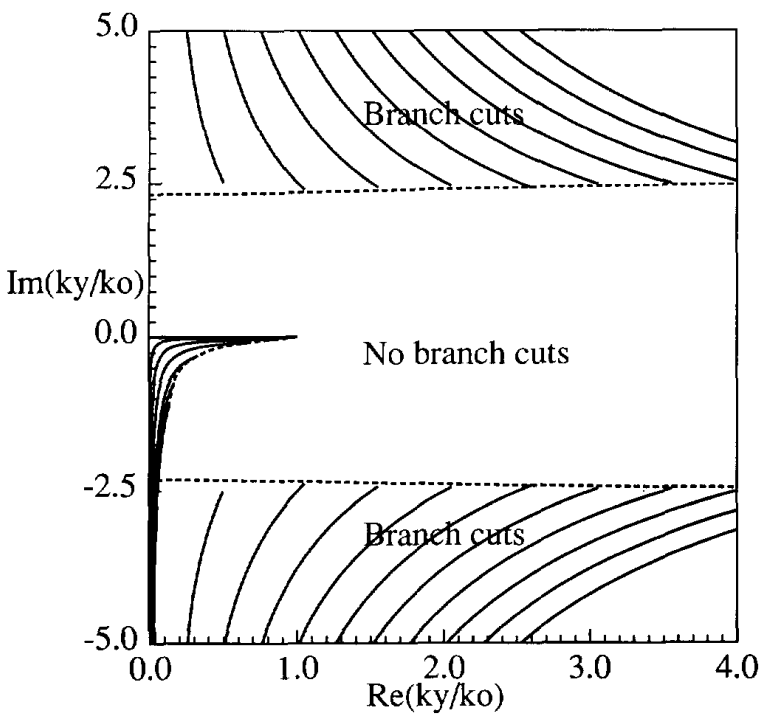

(b)

Fig. 5. Branch cuts. (a) In the complex $k_{x}$. (b) In the complex $k_{y}$ plane.

In order to minimize the computational effort, the basis and test functions are divided into a $k_{x}$-dependent part and a $k_{y}$ dependent part. These parts are calculated once per $k_{x}\left(k_{y}\right)$ integration point. $\tilde{G}_{x x}\left(k_{x}, k_{y}\right)$ is calculated once per $\left(k_{x}, k_{y}\right)$.

$$
Z_{i}\left(k_{y}\right)=\left\{\begin{array}{cc}
j \frac{1}{g} e^{j A \omega_{i}} \int_{0}^{1} \tilde{S}\left(A-j \frac{1}{g} \log z, k_{y}\right) \tilde{A}_{i}\left(A-j \frac{1}{g} \log z\right) z^{\left(\omega_{i} / g\right)-1} d z & \text { for } \omega_{i}>0 \\
\int_{0}^{1 / A} \tilde{S}\left(\frac{1}{v}, k_{y}\right) \tilde{A}_{i}\left(\frac{1}{v}\right) \frac{1}{v^{2}} d v & \text { for } \omega_{i}=0 \\
-j \frac{1}{g} e^{j A \omega_{i}} \int_{0}^{1} \tilde{S}\left(A+j \frac{1}{g} \log z, k_{y}\right) \tilde{A}_{i}\left(A+j \frac{1}{g} \log z\right) z^{-\left(\omega_{i} / g\right)-1} d z & \text { for } \omega_{i}<0
\end{array}\right\} .
$$

$$
g>0
$$




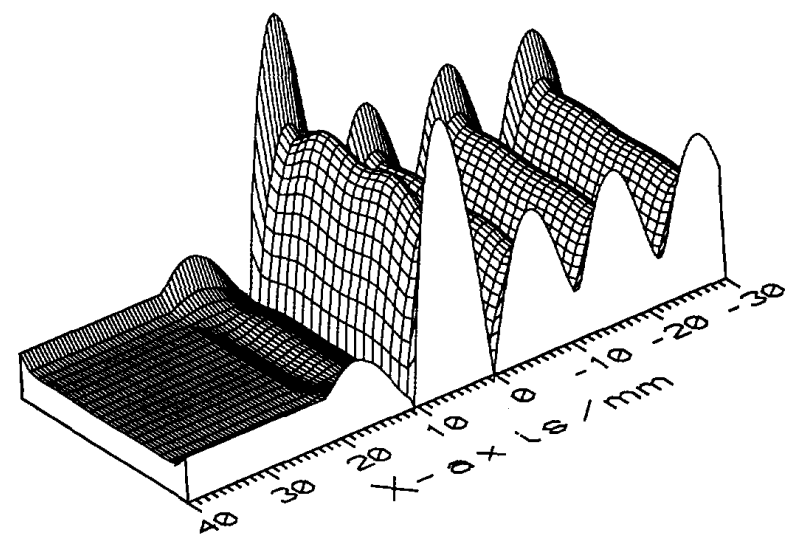

Fig. 6. Current distribution at first resonance. Input parameters: Substrate: $\mathrm{RT} /$ duroid $5870, \mathrm{~d}=0.7874 \mathrm{~mm}, \epsilon_{r}=2.33$ Dimension of the filter: $W=2.30 \mathrm{~mm}, G=0.3 \mathrm{~mm}, L=10 \mathrm{~mm}$. Frequency $=9.66 \mathrm{GHz}$ Basis functions: $(41,41,41) * 3$.

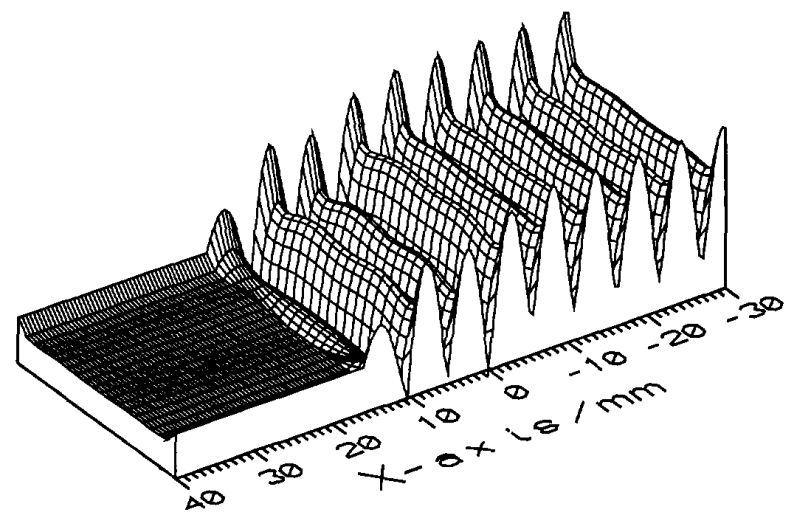

Fig. 7. Current distribution at second resonance. Input parameters are the same as used in Fig. 6 except for the frequency. Frequency $=19.32 \mathrm{GHz}$.

\section{RESULTS}

Using the described procedure for computation of the elements in (31), the current distribution on the filter has been determined from solving the linear equations. In Fig. 5, 6 and 7 , the current distribution at the first and second resonance frequency are shown. The value of the current distributions at the edges are not shown due to the edge singularity. The number of basis functions used for modeling the filter currents are written using the notation $(x, y, z)^{*} v$. The $x, y$ and $z$ denote the number of PWS basis functions used for modeling the current on the input, centre and output microstrip line respectively. $v$ denotes the number of Maxwellian basis function used for the $y$-dependence. Regardless of the number of PWS basis functions, their base covers the area indicated in Fig. 2.

In Figs. 6 and 7, a standing wave at the semi-infinite input microstrip line and a transmitted wave at the semi-infinite output microstrip line can be observed. The excited currents on the centre microstrip line demonstrates the first and second resonance. Since the $x$ directed current is singular at the edges parallel to the $x$ axis (10), the current shown in Figs. 5, 6 and 7 does not include the current value at the edges.

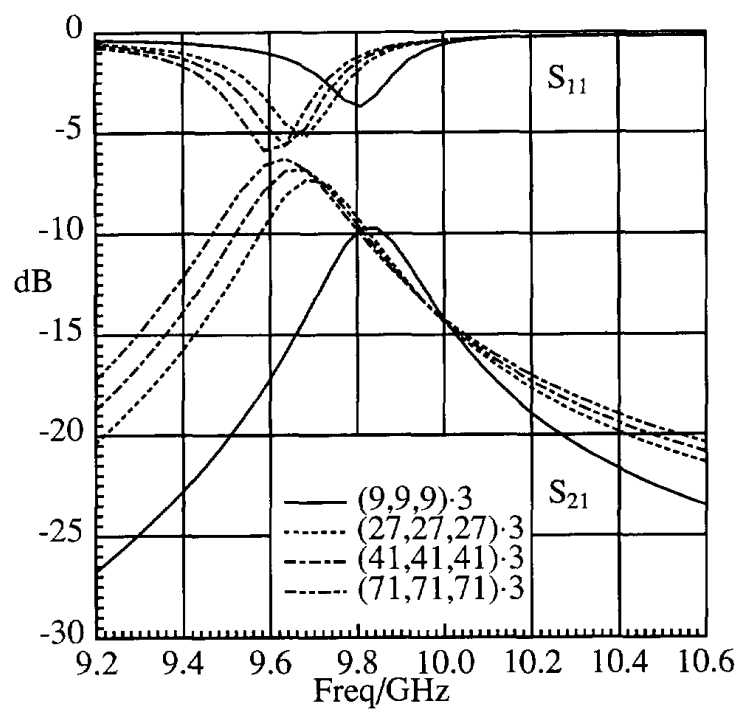

Fig. 8. Amplitude of the $S_{11}$ and $S_{21}$ parameters for the microstrip filter. Input parameters: Substrate: RT/duroid $5870, \mathrm{~d}=0.7874 \mathrm{~mm}, \epsilon_{r}=2.33$ Dimension of microstrip filter: $W=2.30 \mathrm{~mm}, G=0.3 \mathrm{~mm}, L=10 \mathrm{~mm}$.

In Fig. 8, the amplitude of $S_{11}$ and $S_{21}$ are shown. Four sets of curves corresponding to $(9,9,9) * 3,(27,27,27) * 3$, $(41,41,41)^{* 3}$ and $(71,71,71)^{* 3}$ PWS basis functions are shown. From Fig. 8 it is seen that the convergence of the $S$ parameters is dependent of the number of PWS basis functions used for the computations.

This dependence is due to the slope of the current expansion functions at the gaps being limited by the PWS functions used for the expansion. Clearly, increasing the number of PWS modes increases the possible slope of the current at the gaps, resulting in a better approximation to the true current.

The computations are shown together with measurements in Fig. 9. The $\left|S_{11}\right|$ and $\left|S_{21}\right|$ parameters have been measured using a HP8510 network analyzer from which the power lost to surface waves, radiation and losses in the dielectric and conductors are computed. We observe a $0.85 \%$ deviation of the computed resonance frequency and a maximum deviation of $0.6 \mathrm{~dB}$ of the $\left|S_{11}\right|$ and $\left|S_{21}\right|$ parameters at resonance.

To obtain improved numerical results without increasing the number of basis functions, it is necessary to use a new type of basis function in proximity to the gaps which allows for the true slope of the current at the gap edge. Investigations of this type are underway.

\section{CONCLUSION}

A full-wave analysis has been presented for the problem of microstrip gap discontinuities. The $S_{11}$ and $S_{21}$ parameters of the two gap microstrip filter have been computed numerically and compared with measurements. The agreement is excellent. Plots of the current distribution and the parameters have been presented. The spectral integral has been computed using a new integration algorithm which is both fast and accurate. The method is efficient in terms of the number of integration points needed for convergence. The basis and test functions are 


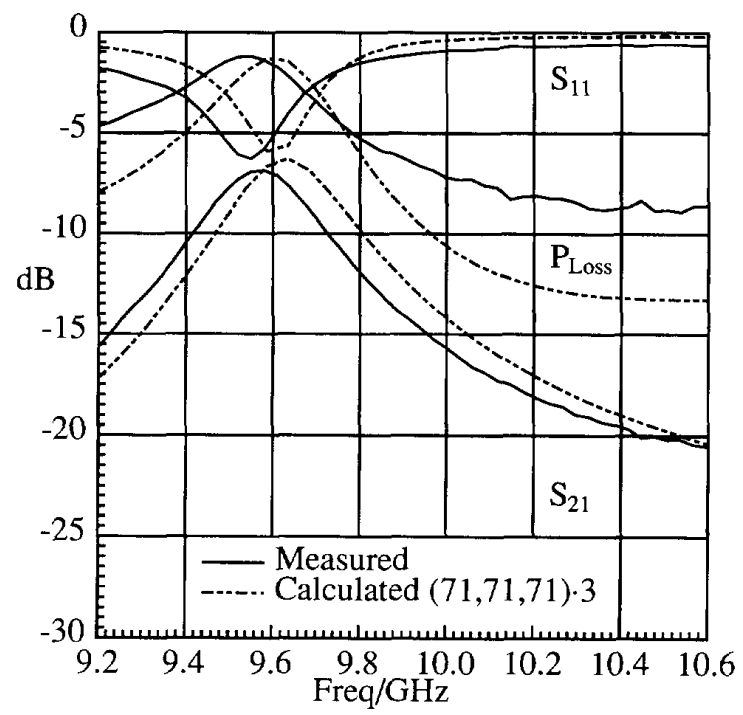

(a)

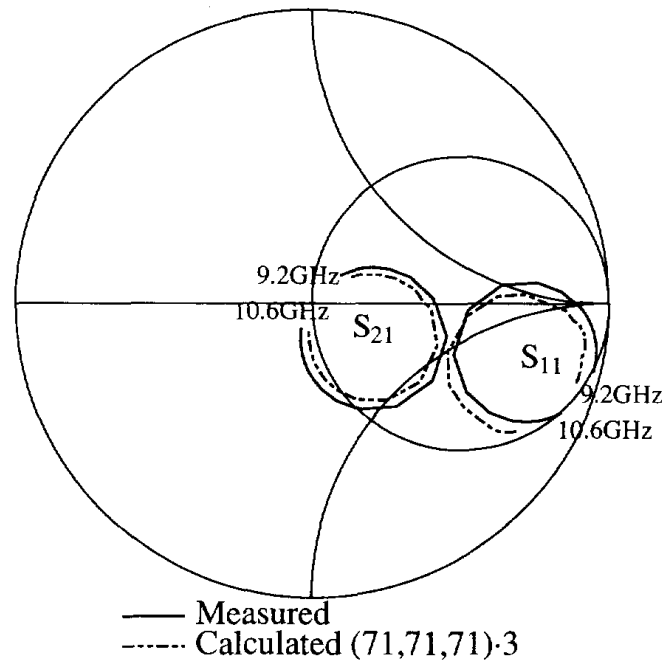

(b)

Fig. 9. Measured and computed amplitude of the $S_{11}$ and $S_{21}$ parameters. (a) Amplitude plot of the measured and computed $S$-parameters and power loss. (b) Measured and computed S-parameters in Smith chart. Input parameters: Substrate: RT/duroid $5870, d=0.7874 \mathrm{~mm}, \epsilon_{r}=2.33$ Dimension of the filter: $W=2.30 \mathrm{~mm}, G=0.3 \mathrm{~mm}, L=10 \mathrm{~mm}$. Frequency sweep $9.2 \mathrm{GHz}$ to $10.6 \mathrm{GHz}$.

divided into a $k_{x}$ and a $k_{y}$-dependent part which are calculated once per $k_{x}\left(k_{y}\right)$ value. The Green's functions are calculated once per $\left(k_{x}, k_{y}\right)$ value. Using this procedure, the $S_{11}$ and $S_{21}$ parameters of the two gap microstrip filter have been computed using 641 basis functions.

\section{ACKNOWLEDGMENT}

The authors would like to thank Juan R. Mosig for introducing us to his method for integration of oscillating functions, i.e. the weighted average method, and Jerzy Lemanczyk for a critical reading of the manuscript.

\section{REFERENCES}

[1] R. W. Jackson and D. M. Pozar, "Full-wave analysis of microstrip openend and gap discontinuities," IEEE Trans. Microwave Theory Tech., vol. MTT-33, no. 10, pp. 1036-1042, Oct. 1985.

[2] H. -Y. Yang, A. Nakatani and J. A. Castañeda, "Efficient evaluation of spectral integrals in the moment method solution of microstrip antennas and circuits," IEEE Trans. Antennas Propagat., vol. 38, no. 7, pp. 1127-1130, July 1990.

[3] J. R. Mosig and F. E. Gardiol, "Microstrip radiation model," Advances in Electronics and Electron Physics, vol. 59, pp. 194-199, 1982.

[4] E. J. Denlinger, "A frequency dependent solution for microstrip transmission lines," IEEE Trans. Microwave Theory Tech, vol. MTT-19, no. 1, pp. 30-39, Jan. 1971

[5] A. Østergaard, "Dyadic Green's functions in the spectral domain," Tech. Rep. R459, Electromagnetics Institute, Technical University of Denmark, Mar. 1991.

[6] D. M. Pozar, "Input impedance and mutual coupling of rectangular microstrip antennas," IEEE Trans. Antennas Propagat., vol. AP-30, no. 6, pp. 1191-1196, Nov. 1982.

[7] T. Itoh, Numerical Techniques for Microwave and Millimeter-Wave Passive Structures. New York: Wiley, 1989.

[8] U. V. Gothelf, "Spectral domain analysis of microstrip circuit elements," E437, M.Sc. thesis, Electromagnetics Institute, Technical University of Denmark, Mar. 1991 (in Danish).

[9] L. Rexberg, "Radiation from planar antennas in the presence of layered dielectric media," Technical Rep. No. 214, Ph.D. thesis, Division of network Theory, Chalmers University of Technology, Gothenborg, Sweden, Apr. 1991.

[10] E. H. Newman and D. Forrai, "Scattering from a microstrip patch," IEEE Transactions on Antennas and Propagation, vol, AP-35, no. 3, pp. 245-251, Mar. 1987.

[11] N. K. Uzunoglu, N. G. Alexopoulos, and J. G. Fikioris, "Radiation Properties of Microstrip Dipoles, IEEE Trans. Antennas Propagation, vol. AP-27, no. 6, pp. 853-858, Nov. 1979.

[12] E. T. Copson, An Introduction to the Theory of Functions of a Complex Variable, London: Oxford University Press, Amen House, E.C.4, 1955, 1957 , ch. 4.

[13] G. N. Watson, Theory of Bessel Functions, 2nd ed., Cambridge: University Press 1962

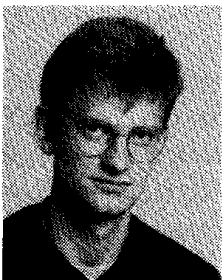

Ulrich V. Gothelf was born in Denmark, on Novem ber 21,1965 . He received the M.Sc.E.E. degree from the Technical University in Denmark in March 1991. He is currently pursuing a Ph.D. degree in electrical engineering at Electromagnetics Institute at the Technical University in Denmark. His main research interest is numerical calculation on $\mathrm{mi}$ crostrip antennas.

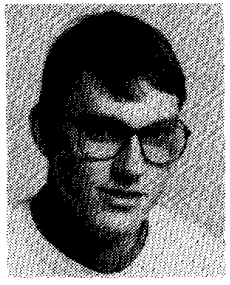

Allan Østergaard was born in Denmark, on May 10,1963 . He received the M.Sc.E.E. degree from the Technical University of Denmark (TUD) in 1987. In 1987 he joined the Electromagnetics Institute at TUD where he developed high speed digital circuitry for the Danish Airborne SAR system. In parallel with the above work he began a Ph.D. program in September 1988 at TUD. His research interests are in the field of numerical analysis and design of radiating printed structures. 